\title{
Alternating Leg Muscle Activation During Sleep and Arousals: A New Sleep-Related Motor Phenomenon?
}

\author{
Ronald D. Chervin, MD, MS, ${ }^{*}$ Flavia B. Consens, MD, and Ekrem Kutluay, MD \\ Sleep Disorders Center, Department of Neurology, University of Michigan, Ann Arbor, Michigan, USA
}

\begin{abstract}
We describe a quickly alternating pattern of anterior tibialis activation, recorded during nocturnal polysomnography in 16 patients. Polysomnography, usually for sleep-disordered breathing, included surface electromyograms over the anterior tibialis of each leg. Cases were identified from approximately 1,500 studies reviewed in the course of standard clinical care. Patients were 12 men and 4 women (mean age, $41 \pm 15$ years; range, 12-70 years). Brief activation of the anterior tibialis in one leg alternated with similar activation in the other leg. Activations occurred at a frequency of approximately 1 to $2 \mathrm{~Hz}$, each lasted between 0.1 and 0.5 seconds, and sequences of alternating activations usually lasted between several and 20 seconds. The phenomenon occurred in all sleep stages but
\end{abstract}

particularly during arousals. Ten of the 16 patients had periodic leg movements during sleep at a rate $\geq 5.0$ per hour, and 12 of the 16 patients were taking antidepressant medication. Alternating leg muscle activation (ALMA) during sleep, at this relatively high frequency, may be a newly described phenomenon. We speculate that ALMA could represent transient facilitation of a spinal central pattern generator for locomotion, perhaps due to serotonergic effects of antidepressant medication. (C) 2003 Movement Disorder Society

Key words: sleep; nocturnal myoclonus syndrome; serotonin uptake inhibitors; polysomnography; locomotion; alternating leg muscle activation
Surface electromyography of the anterior tibialis is commonly performed during polysomnography, mainly to record periodic leg movements during sleep (PLMS). ${ }^{1,2}$ The PLMS can occur on one side or the other at different times of the night, and sometimes simultaneously on both sides but at different rates. ${ }^{3}$ Recent detailed investigation of 10 patients showed that sometimes PLMS alternate sides. ${ }^{4}$ Involvement of upper limbs in some patients has led some authors to speculate that PLMS are spinal fragments of a locomotor pattern, perhaps dysinhibited by supraspinal mechanisms, ${ }^{5}$ but PLMS are typically separated by 20 - to 40 -second intervals that are much longer than those between leg movements during ambulation.

\footnotetext{
*Correspondence to: Ronald D. Chervin, M.D., M.S., Michael S. Aldrich Sleep Disorders Laboratory, University Hospital 8D8702, Box 0117, 1500 East Medical Center Drive, Ann Arbor, MI 48109-0117. E-mail: chervin@umich.edu

Received 20 December 2001; Revised 24 September 2002; Accepted 18 October 2002
}

We describe a sleep-related pattern of alternating leg muscle activation (ALMA) that approximates, in frequency, that seen in human locomotion. Most of the 16 patients who demonstrated ALMA were studied for obstructive sleep apnea, but most also proved to have PLMS, and most also took antidepressant medication.

\section{SUBJECTS AND METHODS}

\section{Subjects}

Subjects were referred to the sleep laboratory and studied between February of 2000 and August of 2001. The 16 index cases of ALMA included in this report were identified from an estimated 1,500 polysomnograms that were reviewed by the authors during the given time period as part of their routine clinical work. After identification of the first case, the search for additional examples was informal, and some cases may have been missed. Data were obtained from sleep studies, referrals, sleep clinic notes, and technician intakes that on the night of polysomnography recorded current medications. Data were examined, correlates identified, and etiological hypotheses generated only after the entire series had been assembled. In addition, medication regimens of identi- 
TABLE 1. Demographics, polysomnography results, medications, and sleep stage in which alternating leg muscle activation was observed

\begin{tabular}{|c|c|c|c|c|c|c|c|c|c|}
\hline Age (yr) & Sex & Study type & Dx & PLMI & AHI & $\mathrm{mO} 2$ & Antidepressant & Other medications & Stage \\
\hline 21 & $\mathrm{~F}$ & Diagnostic & PLMS & 27.3 & 7.7 & 93 & Paroxetine & & 2 \\
\hline 38 & $\mathrm{~F}$ & Titration & OSA & 17.9 & & & Bupropion & $\begin{array}{l}\text { Methylphenidate, } \\
\text { levothyroxin }\end{array}$ & $1,2, \mathrm{REM}$ \\
\hline 14 & $\mathrm{~F}$ & Diagnostic & PLMS & 15.5 & 0.4 & 95 & $\begin{array}{l}\text { Imipramine, } \\
\text { trazodone }\end{array}$ & Buspirone & $2,3, \mathrm{REM}$ \\
\hline 12 & M & Diagnostic & $\begin{array}{l}\text { PLMS, } \\
\text { enuresis }\end{array}$ & 2.2 & 1.2 & 94 & Fluoxetine & $\begin{array}{l}\text { Montelukast, prednisone, } \\
\text { inhaler }\end{array}$ & $1,2, \mathrm{REM}$ \\
\hline 46 & M & Diagnostic & OSA & 0.0 & 110.2 & 86 & Fluoxetine & $\begin{array}{l}\text { Pramipexole, atorvastatin, } \\
\text { omeprazole, } \\
\text { clarithromycin, } \\
\text { clonazepam prn, } \\
\text { vitamin B12 injections }\end{array}$ & 1,2 \\
\hline 37 & M & Diagnostic & OSA, PLMS & 13.8 & 10.4 & 90 & Bupropion & Atorvastatin & 1,2 \\
\hline 57 & M & Diagnostic & $\begin{array}{l}\text { OSA, } \\
\text { bruxism }\end{array}$ & 3.7 & 20.3 & 90 & $\begin{array}{l}\text { Amitriptyline, } \\
\text { valproic } \\
\text { acid }\end{array}$ & $\begin{array}{l}\text { Atorvastatin, aspirin, } \\
\text { vitamin } C \text {, vitamin } E\end{array}$ & $1,2, \mathrm{REM}$ \\
\hline 36 & M & Split night & OSA & 3.5 & 10.8 & 82 & Venlafaxine & $\begin{array}{l}\text { Dextroamphetamine \& } \\
\text { racemic amphetamine }\end{array}$ & $1,2, \mathrm{REM}$ \\
\hline 46 & M & Diagnostic & PLMS & 26.4 & 1.9 & 91 & Paroxetine & Sildenafil prn & 2 \\
\hline 44 & M & Diagnostic & OSA, PLMS & 53.2 & 7.9 & 86 & None & $\begin{array}{l}\text { Phenobarbital, phenytoin, } \\
\text { aspirin, loratadine }\end{array}$ & REM \\
\hline 53 & $\mathrm{~F}$ & Titration & OSA & 4.9 & & & $\begin{array}{l}\text { Venlafaxine, } \\
\text { trazodone, } \\
\text { nortriptyline }\end{array}$ & $\begin{array}{l}\text { Estrogen, } \\
\text { medroxyprogesterone } \\
\text { acetate, rofecoxib, } \\
\text { atorvastatin, triamterene } \\
\text { \& hydrochlorothiazide, } \\
\text { loratadine \& } \\
\text { pseudoephedrine, } \\
\text { montelukast, salmeterol, } \\
\text { tamsulosin, albuterol }\end{array}$ & 1,2 \\
\hline 44 & M & Titration & OSA & 8.1 & & & None & Ibuprofen & 1 \\
\hline 70 & M & Titration & OSA, PLMS & 48.6 & & & None & $\begin{array}{l}\text { Lisinopril, pioglitazone, } \\
\text { metformin, atorvastatin, } \\
\text { aspirin; nine vitamins } \\
\text { and supplements }\end{array}$ & 1,2 \\
\hline
\end{tabular}

PLMI, periodic leg movement index; AHI, apnea hypopnea index (for diagnostic studies only); mO2, minimum oxygen saturation (for diagnostic studies only); Stage, sleep stages (i.e., other than wakefulness) from which ALMA were observed to emerge; Diagnostic, baseline sleep study, without intervention; Titration, titration study to determine appropriate setting for continuous positive airway pressure (CPAP); Split Night, study in which the first portion of the night was a diagnostic study and the second portion was used for CPAP titration; OSA, obstructive sleep apnea; PLMS, periodic leg movements during sleep; REM, rapid eye movement sleep; 1, 2, and 3: non-REM sleep stages 1, 2, and 3/4, respectively.

fied cases were compared to those of 100 patients within the same age range who were referred to the sleep laboratory during the same period. These 100 subjects were the first 10 to be studied in each of 10 consecutive months between October of 2000 and July of 2001 .

The mean age of the index subjects was $41 \pm 15$ (SD) years, and $12(75 \%)$ were men (Table 1$)$. Twelve had been referred for evaluation of sleep-disordered breathing or for continuous positive airway pressure (CPAP) titration to identify an effective setting for home use. One subject had been referred for assessment of each of the following: possible narcolepsy, PLMS, sleepwalking, and movement during sleep in the context of Hunting- ton's disease. Nine subjects complained of excessive daytime sleepiness or fatigue, and 7 had Epworth Sleepiness Scale scores that were greater than $10 .^{6}$ Clinic or lab notes showed complaints of insomnia in 2 subjects. Four subjects had a complaint of restless legs or more general restlessness at night, but none had a bed partner who had mentioned involuntary leg kicking. One subject complained of sudden nocturnal muscle contractions in the legs and the sensation that his legs were vibrating.

Of the 16 subjects, 10 had had a history and physical examination performed by a board-certified or eligible neurologist, 2 by neurosurgeons, 2 by internists, and 1 by a physician's assistant. No recorded examination was 
available for 1 index subject. Aside from the patient with Huntington's disease, none of the remaining 14 patients with history and physical data had a history of Parkinson's disease, and none were noted to have the resting tremor, bradykinesia, rigidity, or postural instability that characterize this condition.

All subjects except for 3 had an apnea/hypopnea index (AHI, number of events per hour of sleep) $\geq 5$ on the current or previous diagnostic study. The periodic leg movement index (PLMI) was $\geq 5$ in $10(63 \%)$ of the index studies; only 2 patients had no scored PLMS. Two patients without PLMI $\geq 5$ had many hypopneas and arousals associated with leg movements that would have counted as PLMS had hypopneas not been scored. Four patients met criteria for restless legs syndrome; 10 did not, and 2 did not have sufficient information available to make this determination adequately. ${ }^{7}$ No subject demonstrated epileptiform activity on the limited electroencephalogram (EEG) included in the polysomnographic montage. Demographics, diagnoses at the time that polysomnograms were interpreted, PLMI, AHI, minimum oxygen saturation, antidepressants taken at the time of the study, and other medications are listed in Table 1. Twelve of the 16 index patients $(75 \%)$ were taking antidepressants at the time of polysomnography. In contrast, among the 100 comparison subjects referred for polysomnography during a similar time interval, only 22 (22\%) were taking antidepressants. Listed reasons for antidepressants among the index cases were depression $(\mathrm{n}=7)$, bipolar disorder $(\mathrm{n}=1)$, anxiety disorder $(\mathrm{n}=$ $1)$, smoking cessation $(n=1)$, attention-deficit/hyperactivity disorder $(\mathrm{n}=1)$, and behavioral control in the patient with Huntington's disease $(\mathrm{n}=1)$.

\section{Polysomnography}

Polysomnograms included two central and two occipital EEG leads, chin surface electromyogram (EMG), two electro-oculogram (EOG) leads, left and right anterior tibialis surface EMG leads, two electrocardiogram (EKG) leads, nasal and oral airflow (thermocouples in diagnostic studies, nasal and oral pressure in titration studies), chest and abdominal excursion (piezoelectric strain gauges), and oximetry. Among the 16 index studies that came to the authors' attention, nine recordings were made for diagnostic purposes, six were made during continuous positive airway pressure (CPAP) titration studies, and one was a split-night study in which the first portion was diagnostic and the second was used to titrate CPAP.

Records were scored by experienced technicians who had scored at least $90 \%$ of a set of validity studies correctly. Apneas were defined as 10 seconds of absent airflow. Hypopneas were scored as 10-second decrements in airflow, chest, or abdominal movement followed by an arousal, awakening, or oxygen desaturation of four or more percentage points. The PLMS were scored when they met criteria for duration ( 0.5 to 5 seconds), periodicity (5 to 120 seconds between each movement), and number (three or more in a row). When a hypopnea occurred between two anterior tibialis activations that would otherwise qualify to be scored as PLMS, the event was scored as a hypopnea rather than two PLMS.

\section{RESULTS}

\section{ALMA}

The ALMA pattern was observed in each subject, usually several times in each recording (Fig. 1). Defined as more than four discrete and alternating muscle activations with $\leq 2$ seconds between activations, the number of ALMA sequences during sleep per record ranged from 1 to 40 (mean $\pm \mathrm{SD}, 13 \pm 12$ ). Each sequence of ALMA during sleep lasted for 1.4 to 22.2 seconds, and each was composed of alternating anterior tibialis activations of approximately 0.1 to 0.5 seconds in length. These sequences sometimes began with one to several lengthy activations (1-2 seconds) in one or both legs. The rate of leg activation was usually between 1 and 2 $\mathrm{Hz}$, but ranged from 0.5 to $3.0 \mathrm{~Hz}$. Some patients showed similar activity in one leg, at times, rather than two (Fig. 2A).

In 12 subjects, ALMA was seen to closely precede or follow an arousal or awakening and to gradually diminish as the patient returned to sleep (Fig. 3). However, the phenomenon was seen without arousals in 4 subjects. With or without arousals, ALMA was observed to arise from non-rapid eye movement (non-REM) sleep stages $1,2,3$, and 4, and from REM sleep. Leg muscle activations during REM sleep (Fig. 2B) were often briefer and somewhat less regular than during non-REM sleep (Fig. $2 \mathrm{~A}$ ), but could still be distinguished from the irregular, nonalternating phasic twitches that normally characterize REM sleep (Fig. 2C). When arousals did occur, they were usually observed to start before ALMA ( $\mathrm{n}=7$ subjects). In 2 subjects, arousals came either before or after ALMA, and in 3 subjects, arousals consistently followed the onset of ALMA.

In some cases, 5- to 10-second sequences of ALMA recurred at intervals that resembled those between PLMS (e.g., 20-30 seconds). However, sequences of ALMA also occurred just before, just after, or occasionally distinctly between PLMS without any obvious tendency to reset the periodicity of the PLMS. In 1 subject, a record- 


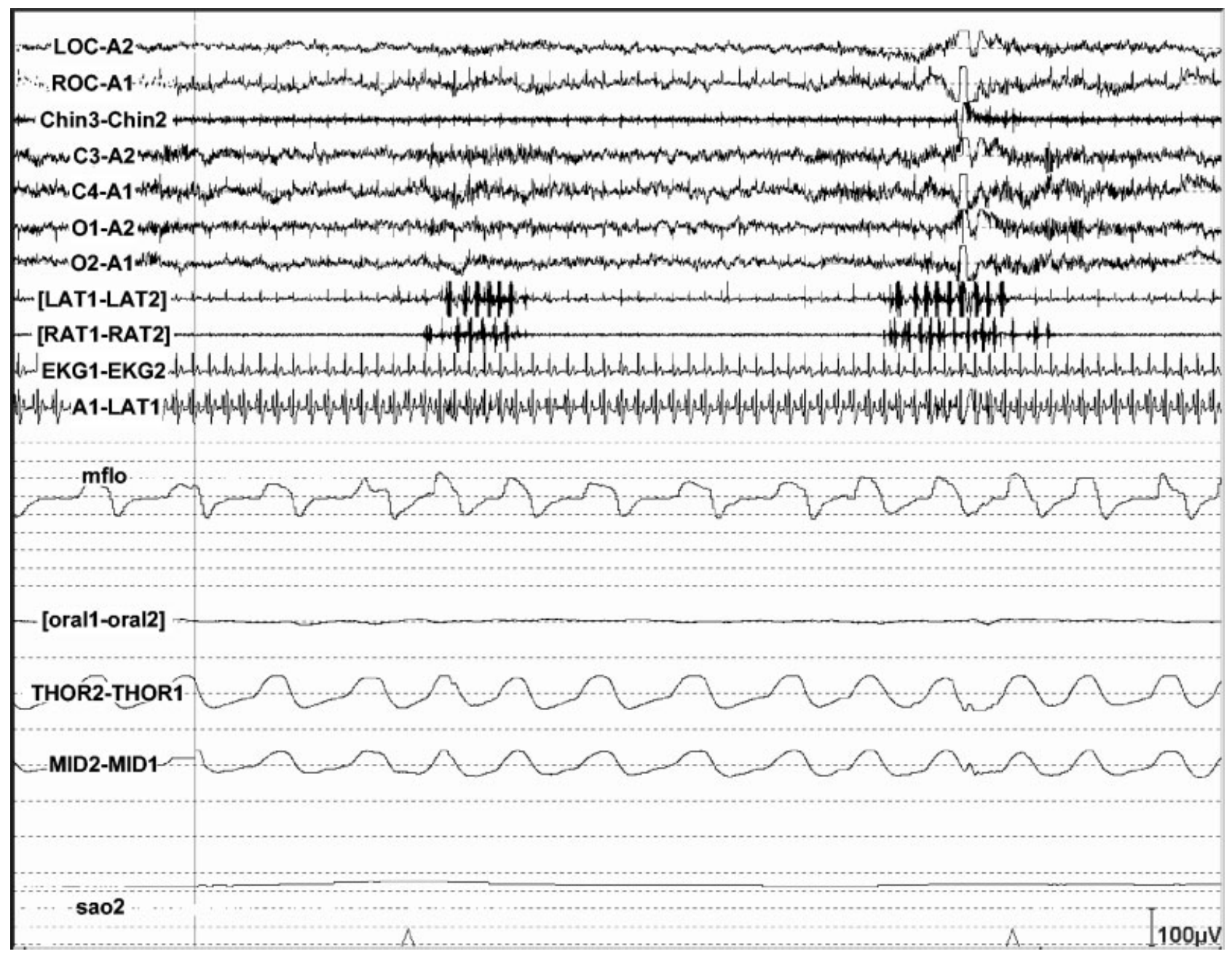

FIG. 1. Sequences of alternating leg muscle activation (ALMA) in a 53-year-old woman, during arousals from this 60-second segment of stage 1 sleep. LOC-A2, ROC-A2 = left and right electro-oculogram, respectively; Chin3-Chin2 = chin surface electromyogram; C3-A2, C4-A1, O1-A2, O2-A1 = central and occipital derivations of international 10-20 electrode placement system; LAT1-LAT2, RAT1-RAT2 = left and right anterior tibialis surface electromyogram; EKG1-EKG2, A1-LAT1 = two electrocardiogram leads; mflo = mask airflow during continuous positive airway pressure titration study; oral 1 -oral $2=$ oral leak as revealed by thermocouple; THOR2-THOR1 $=$ thoracic excursion; MID2-MID1 = abdominal excursion; sao2 $=$ oxygen saturation $(\%)$.

ing of extensor forearm muscle surface EMG was also performed. The results suggested some upper extremity alternating activity in association with ALMA, although less clearly defined than the lower extremity phenomenon (Fig. 4).

\section{Repeat Recordings}

The index studies that demonstrated ALMA were collected during a 19-month period, during which less than half of the laboratory's studies were reviewed by the authors. Among the 9 patients who had diagnostic index studies, 3 later had CPAP titration studies, and review of these studies showed ALMA in 2. Among the 6 patients whose index studies were CPAP titrations, all 6 had had diagnostic studies in the same laboratory, and each of these diagnostic studies also showed ALMA. For the 9 subjects who had both diagnostic and CPAP titration studies, the mean number of ALMA sequences in the diagnostic studies was 14, and that in the titration studies was also 14. The relationship between the timing of arousals and ALMA was also preserved between studies. Among patients who had a repeat recording, the 1 patient whose ALMA preceded arousals in the index study showed the same pattern during the repeat study, and the 5 in whom ALMA followed arousals also showed the same pattern in the repeat studies.

\section{DISCUSSION}

We describe a previously unreported pattern of alternating leg muscle activation, at a rate of approximately 1 


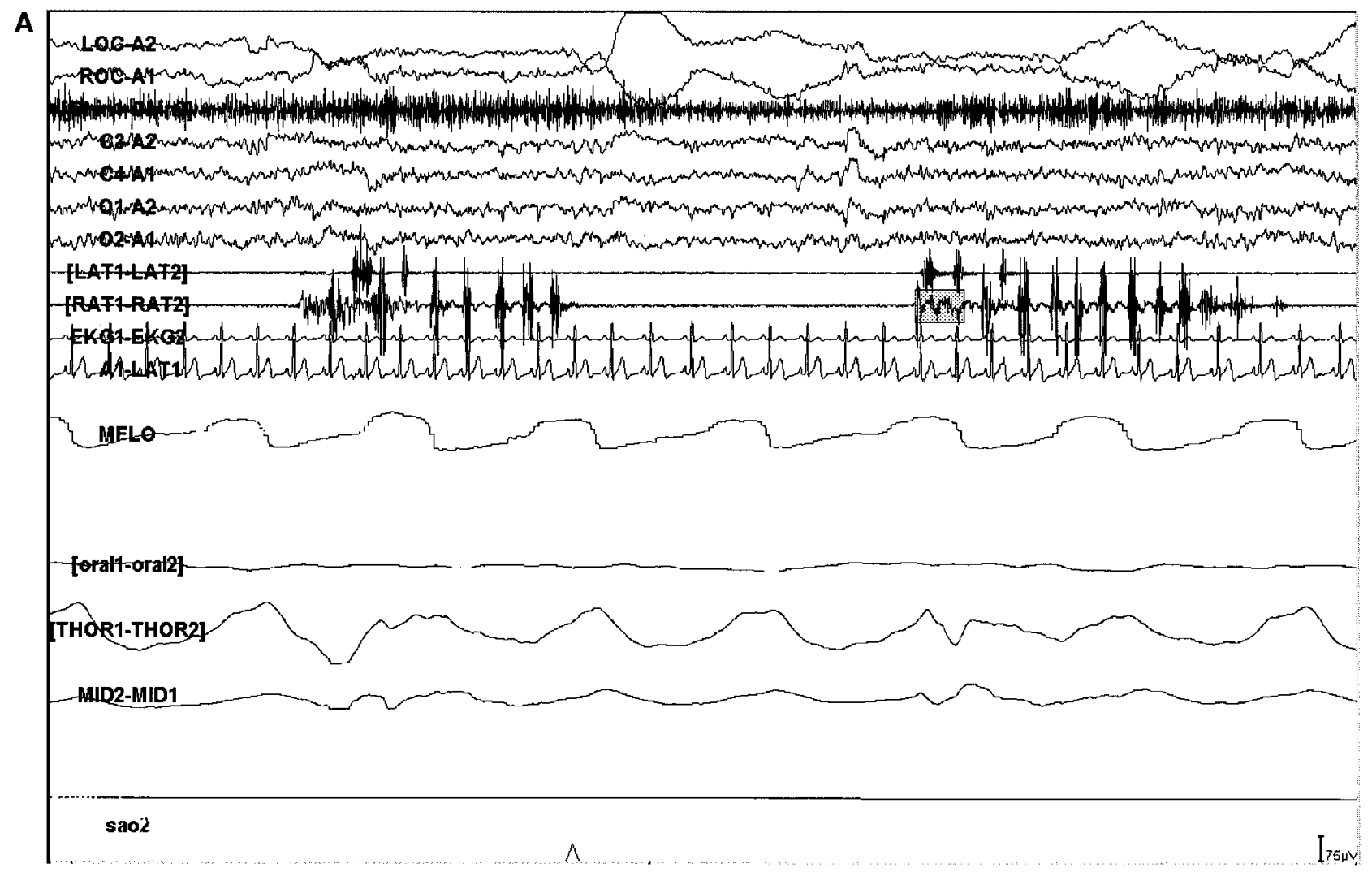

FIG. 2. Repeated sequences of alternating leg muscle activation (ALMA) in a 38-year-old woman (A,B). In A, some right leg activations are not followed by left leg activations during 30 seconds of stage 1 non-rapid eye movement (non-REM) sleep. In $\mathbf{B}$, activations are shorter and no associated arousal occurs during 30 seconds of REM sleep. C: In contrast, a recording from a 36-year-old man without ALMA shows the irregular, nonalternating pattern of muscle activity that normally characterizes REM sleep. Time scale and abbreviations as in Figure 1.

to $2 \mathrm{~Hz}$, during sleep or arousals from sleep. The majority of the 16 patients with ALMA had obstructive sleep apnea, PLMS, and medication regimens that included one or more antidepressants. The ALMA was frequently associated with arousals, but also occurred in every sleep stage. As discussed below, the ALMA could represent any one of several previously reported motor phenomena associated with sleep. Whether ALMA reflects a new, distinct motor phenomenon or an elaboration of a previously defined entity, the question of why its defining characteristic-regular alternation of activation from one leg to the other-has not been described previously is not completely clear. The phenomenon may be rare, and we may have identified it in part because our laboratory performs a large volume of nocturnal studies. Also, in contrast to some sleep laboratories, ours routinely records right and left leg movements independently on two separate channels rather than one combined channel.

The ALMA bears some resemblance to at least six previously described phenomena. PLMS has been described to occur in the form of polymyoclonus-bursts of multiple jerks in frequencies not dissimilar to those of ALMA - although not in an alternating side-to-side manner and not in association with antidepressant medication. ${ }^{1,3}$ The frequency of PLMS in the current patient series $(63 \%)$ exceeds the already elevated frequency (24\%) among patients studied in our laboratory for suspected sleep apnea. ${ }^{8}$ Against the hypothesis that ALMA represents one form of PLMS, however, were several examples where occurrence of ALMA in between clear PLMS had no effect on the periodicity of the PLMS. Furthermore, in contrast to ALMA, each leg activation in PLMS lasts 0.5 to 5.0 seconds and the intervals between movements are at least 4 seconds.

Montagna and colleagues described propriospinal myoclonus in 3 patients with severe insomnia. ${ }^{9}$ Features of propriospinal myoclonus that resemble ALMA include occurrence at transitions from wake to sleep, quasiperiodic intervals of 4 to 30 seconds between movements, and a polyclonic composition of most movements. However, in contrast to ALMA, propriospinal myoclonus is associated with severe insomnia, causes marked complaints of involuntary jerking movements, occurs only 


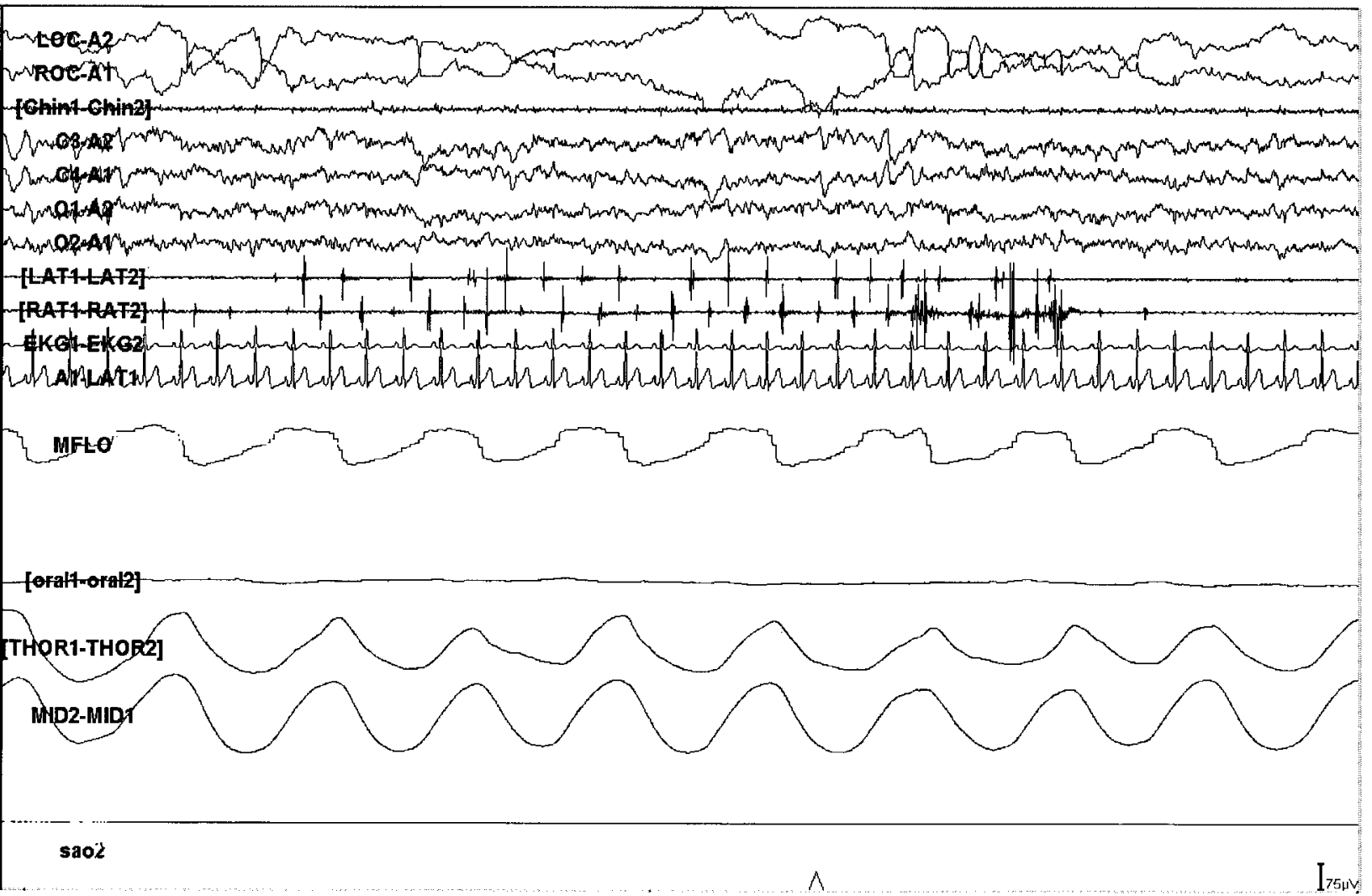

C

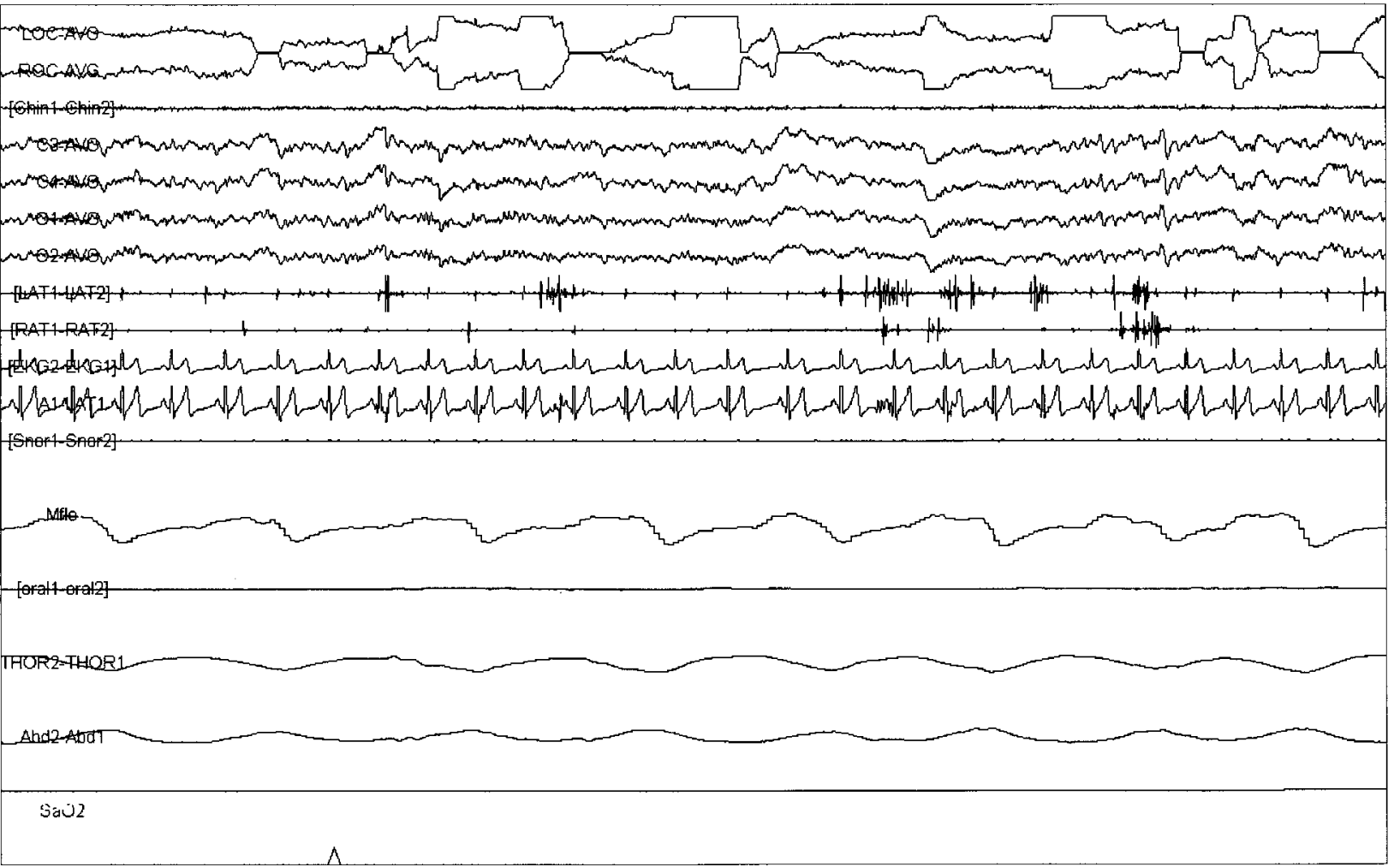

FIGURE 2. (Continued) 


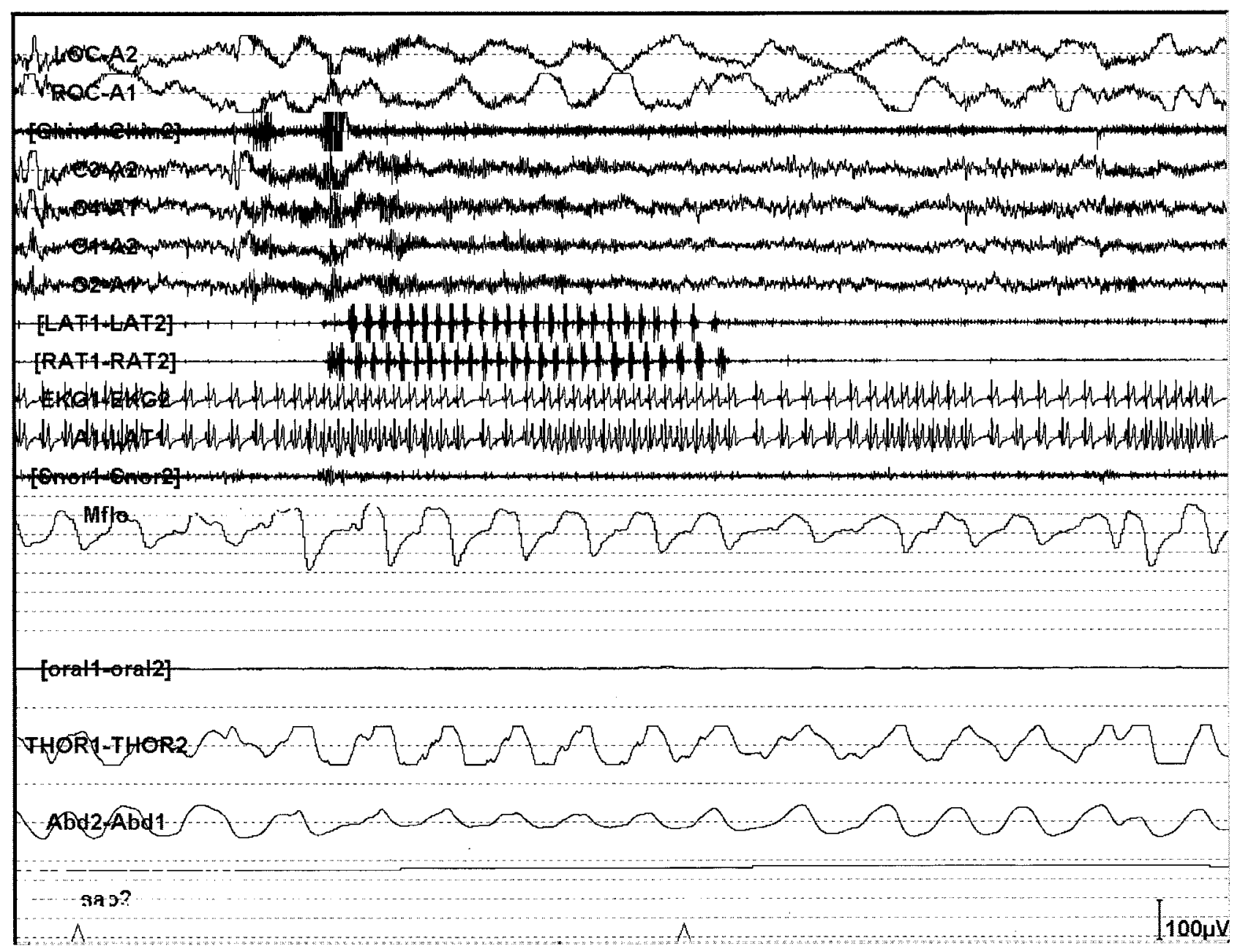

FIG. 3. An alternating leg muscle activation (ALMA) sequence that arose during an arousal and diminished as sleep returned to this 44-year-old man. Time scale and abbreviations as in Figure 1; snore1-snore2 = snoring monitor; ABD1-ABD2 = abdominal excursion.

during wakefulness, shows no reported tendency to alternate between sides, and activates chin muscles (among others) at the same time as the leg muscles.

The rhythmic movement disorder of sleep can occur at rates up to $2 \mathrm{~Hz}$, usually in wakefulness or light sleep but rarely in other stages. ${ }^{1}$ The disorder can rarely involve leg banging rather than the more common head banging or body rocking, and in adults may be associated with psychopathology. Rhythmic movement disorder is sometimes comorbid with PLMS, at least in patients with severe restless legs syndrome, and some authors have hypothesized that a similar pathophysiology may underlie all three conditions. ${ }^{10,11}$ However, rhythmic movement disorder is most common in children, usually occurs for many minutes rather than seconds, does not involve alternating leg movements, and bears no known relation to antidepressant medications.
In Parkinson's disease resting tremor can affect the legs. The tremor usually stops at sleep onset, but can recur for brief intervals during arousals, light non-REM sleep, or occasionally REM sleep. However, the tremor is usually faster $(3-5 \mathrm{~Hz})$ than ALMA and has not been reported to alternate sides. In addition, Parkinson's disease usually begins after age 50 , whereas all but 4 of our 16 patients were under 50 years of age. None of the patients in our series had a known history of Parkinson's disease or showed suggestive signs on available neurological examinations.

Rhythmic leg movements have been described during wakefulness in patients with neuroleptic-induced akathisia. ${ }^{12-14}$ Electromyographic activity can be recorded at the anterior tibialis at 0.5 to $3.0 \mathrm{~Hz}$, for seconds to minutes, but alternation between sides, occurrence during sleep, and potential association with antidepressant agents have not been described. 


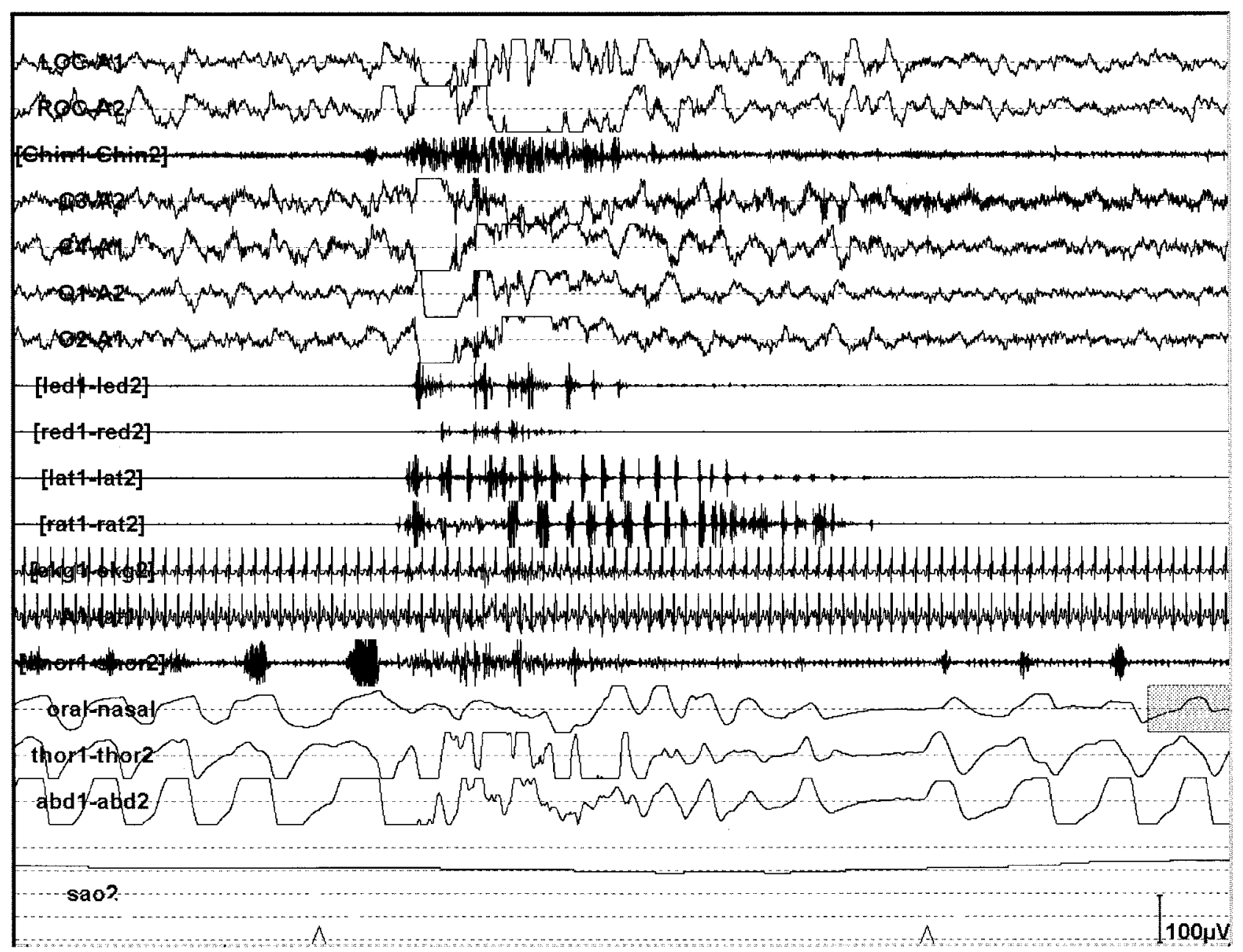

FIG. 4. An alternating leg muscle activation (ALMA) sequence that coincided with similar muscle activations in the arms of this 57-year-old man. Time scale and abbreviations as in Figures 1 and 3; led1-led2, red1-red2 = left and right forearm extensor surface electromyogram; oral-nasal = oral and nasal airflow monitored by thermocouple.

Perhaps of greatest relevance, Broughton described a hypnagogic foot tremor, in 2 patients who complained of insomnia, that resembles ALMA in polysomnographic appearance, rate $(0.5-1.5 \mathrm{~Hz})$, and association with arousals and light sleep. ${ }^{15}$ However, both patients had had recent head trauma (to which the author ascribed the phenomenon), both had more prominent insomnia than we found in our series, neither had PLMS or sleep apnea, and neither showed the tremor in deep non-REM or REM sleep. Alternation of movement from one leg to the other was not mentioned, although the one published polysomnographic fragment suggests alternation may have occurred. ${ }^{15}$ The medications for each patient were not listed, but administration of a tricyclic antidepressant after head trauma is common. A subsequent study identified the phenomenon in 28 subjects among 375 studied: 355 were patients with sleep-related complaints and 20 were healthy control subjects. ${ }^{16}$ The authors called the phenomenon "rhythmic feet movements while falling asleep," although the movements were sometimes observed during light non-REM sleep or arousals from REM sleep. These patients did not have head trauma, most did not have insomnia, most did have sleep apnea, several had PLMS or restless legs syndrome, but only 1 took an antidepressant. The leg movements "occurred generally bilaterally but asynchronous in both legs." However, one figure clearly shows regular alternation between left and right anterior tibialis EMG activations, not commented on by the authors, but indistinguishable from ALMA.

The cause of ALMA remains a mystery, but current observations allow some speculation. The large majority (75\%) of our patients took antidepressants at the time of the study. Antidepressants, including selective serotonin 
reuptake inhibitors (SSRIs) and tricyclic compounds, often worsen restless legs syndrome and PLMS, perhaps because of increased serotonergic and associated decreased dopaminergic transmission. ${ }^{17-19}$ Bupropion differs from SSRIs in that it also inhibits dopamine reuptake; as might be expected, this compound has been reported to reduce PLMS. ${ }^{20}$ Therefore, the observation that 2 patients with ALMA took bupropion and no other antidepressant argues against the idea that ALMA is due to dopaminergic insufficiency or associated PLMS. The possibility remains that increased serotonergic transmission facilitates ALMA, as bupropion also inhibits reuptake of this neurotransmitter.

The rate of ALMA approximates that of leg movements during locomotion, the rhythm for which is mediated in large part by neuronal networks intrinsic to the spinal cord. ${ }^{21}$ Isolated rat spinal cord preparations contain these networks, or central pattern generators, that produce lumbar ventral root output upon exposure to serotonin. ${ }^{22}$ Output alternates side-to-side in a manner and frequency that resembles ALMA. ${ }^{22}$ Serotonin receptors exist at all spinal cord levels, and descending fibers from brainstem serotonergic nuclei synapse on spinal cord motorneurons, where they modulate and generally facilitate motor output. If ALMA during sleep and arousals does reflect locomotor network activity, we speculate that antidepressants might produce ALMA through enhancement of serotonergic transmission to central pattern generators of the spinal cord. The PLMS recorded in many of our patients could represent a related or else similarly facilitated process.

Many potential links between our patients remain unexplored, and we cannot rule out the possibility that one of these explains the motor patterns we observed. However, the highly consistent finding of ALMA in repeated recordings suggests the phenomenon was not an artifact, a transient occurrence, or an acute result of untreated sleep-disordered breathing. Whether the phenomenon may carry any clinical import remains unknown, but its occurrence at sleep-wake transitions raises the possibility that further study might provide insight into mechanisms of motor control during sleep.

Acknowledgments: We thank Dr. Roger Albin for his helpful review of this manuscript and Dr. John W. Winkelman for valuable input on possible etiologies of ALMA.

\section{REFERENCES}

1. American Sleep Disorders Association. International classification of sleep disorders, revised: diagnostic and coding manual. Rochester, MN: American Sleep Disorders Association; 1997.

2. Chesson AL, Ferber RA, Fry JM, Grigg-Damberger M, Hartse KM, Hurwitz TD, Johnson S, Kader GA, Littner M, Rosen G. The indications for polysomnography and related procedures. Sleep 1997;20:423-487.

3. Lugaresi E, Cirignotta F, Montagna P. Nocturnal myoclonus and restless legs syndrome. Adv Neurol 1986;43:295-307.

4. Provini F, Vetrugno R, Meletti S, Plazzi G, Solieri L, Lugaresi E, Coccagna G, Montagna P. Motor pattern of periodic limb movements during sleep. Neurology 2001;57:300-304.

5. Chokroverty S, Jankovic J. Restless legs syndrome: a disease in search of identity. Neurology 1999;52:907-910.

6. Johns MW. A new method for measuring daytime sleepiness: the Epworth Sleepiness Scale. Sleep 1991;14:540-545.

7. Walters AS, the International Restless Legs Syndrome Study Group. Toward a better definition of the restless legs syndrome. Mov Disord 1995;10:634-642.

8. Chervin RD. Periodic leg movements and sleepiness in patients evaluated for sleep-disordered breathing. Am J Respir Crit Care Med 2001;164:1454-1458.

9. Montagna P, Provini F, Plazzi G, Liguori R, Lugaresi E. Propriospinal myoclonus upon relaxation and drowsiness: a cause of severe insomnia. Mov Disord 1997;12:66-72.

10. Walters AS. Frequent occurrence of myoclonus while awake and at rest, body rocking and marching in place in a subpopulation of patients with restless legs syndrome. Acta Neurol Scand 1988;77: $418-421$.

11. Lombardi C, Provini F, Vetrugno R, Plazzi G, Lugaresi E, Montagna P. Pelvic movements as rhythmic motor manifestations associated with restless legs syndrome. Mov Disord 2003;18:110113.

12. Walters AS, Hening W, Rubinstein M, Chokroverty S. A clinical and polysomnographic comparison of neuroleptic-induced akathisia and the idiopathic restless legs syndrome. Sleep 1991;14:339345.

13. Lipinski JF, Hudson JI, Cunningham SL, Aizley HG, Keck PE Jr, Mallya G, Aranow RB, Lukas SE. Polysomnographic characteristics of neuroleptic-induced akathisia. Clin Neuropharmacol 1991; 14:413-419.

14. Cunningham SL, Winkelman JW, Dorsey CM, Lukas SE, Richardson GS, Sholar MB, Hunt A. An electromyographic marker for neuroleptic-induced akathisia: preliminary measures of sensitivity and specificity. Clin Neuropharmacol 1996;19:321-332.

15. Broughton R. Pathological fragmentary myoclonus, intensified "hypnic jerks" and hypnagogic foot tremor: three unusual sleeprelated movement disorders. In: Koella WP, Obal F, Schulz H, Visser P, editors. Sleep '86. Stuttgart: Gustav Fischer Verlag; 1988. p 240-243.

16. Wichniak A, Tracik F, Geisler P, Ebersbach G, Morrissey SP, Zulley J. Rhythmic feet movements while falling asleep. Mov Disord 2001;16:1164-1170.

17. Lipinski JF Jr, Mallya G, Zimmerman P, Pope HG Jr. Fluoxetineinduced akathisia: clinical and theoretical implications. J Clin Psychiatry 1989;50:339-342.

18. Dorsey CM, Lukas SE, Cunningham SL. Fluoxetine-induced sleep disturbance in depressed patients. Neuropsychopharmacology (Berl) 1996;14:437-442.

19. Hargrave R, Beckley DJ. Restless leg syndrome exacerbated by sertraline. Psychosomatics 1998;39:177-178.

20. Nofzinger EA, Fasiczka A, Berman S, Thase ME. Bupropion SR reduces periodic limb movements associated with arousals from sleep in depressed patients with periodic limb movement disorder. J Clin Psychiatry 2000;61:858-862.

21. Grillner S, Cangiano L, Hu GY, Thompson R, Hill R, Wallén P. The intrinsic function of a motor system: from ion channels to networks and behavior. Brain Res 2000;886:224-236.

22. Schmidt BJ, Jordan LM. The role of serotonin in reflex modulation and locomotor rhythm production in the mammalian spinal cord. Brain Res Bull 2000;53:689-710. 